\title{
Finite size scaling in the 2D XY-model and generalized universality
}

\author{
G. Palma, T. Meyer and R. Labbé \\ Departamento de Física, Universidad de Santiago de Chile \\ Casilla 307, Correo 2, Santiago, Chile
}

(Received October 23, 2018)

\begin{abstract}
In recent works [1] (BHP), a generalized universality has been proposed, linking phenomena as dissimilar as $2 \mathrm{D}$ magnetism and turbulence. To test these ideas, we performed a MC study of the $2 D X Y$-model. We found that the shape of the probability distribution function for the magnetization $M$ is non Gaussian and independent of the system size -in the range of the lattice sizes studied- below the Kosterlitz-Thoules temperature. However, the shape of these distributions does depend on the temperature, contrarily to the BHP's claim. This behavior is successfully explained by using an extended finite-size scaling analysis and the existence of bounds for $M$.
\end{abstract}

PACS numbers: 05.10.Ln, 05.50.+q, 64.60.C, 75.30.-m 
The study of critical phenomena is of great interest not only because it allows the understanding of a large number of very different physical systems, like the super fluid Helium three, low temperature superconductors, ferromagnetic-paramagnetic systems, turbulent fluids and plasmas, polymers, snow flakes and earthquakes, but also due to the existence of scale independence of the fluctuations at the critical temperature. In fact, although the underlying inter-molecular forces, responsible for the existence of phase transitions, have a well-defined length scale, the structures they give rise do not. This leads, very close to the critical temperature, to the power-law behavior of physical quantities, which characterizes universality. The main challenge of the theory of critical phenomena is to explain how dissimilar systems exhibit the same critical behavior. Renewed interest in this subject has been raised, because in a seminal paper [1] (BHP), it was argued that turbulence experiments can be explained in terms of a self-similar structure of fluctuations, just as in a finite critical system like the harmonic finite $2 D X Y$-model (2DHXY-model). The starting point of this conjecture was the observation that the probability distribution function (PDF) of the injected power fluctuations in a confined shear turbulent flow [2] has the same shape as the PDF of the magnetization in the $2 D H X Y$-model. It was also proposed that this analogy should provide a new application of finite size scaling in critical systems with experimental consequences.

In this paper, we report the results of a high precision Monte Carlo study of the full $2 D X Y$-model. This computation was carried out over the whole physical range of temperatures. The magnetic susceptibility was computed and the lattice-shifted critical temperature was obtained for different lattice sizes. Scaling laws for the magnetization-temperature ratio were tested. Our results agree with the rigorous findings of Chung [3]. We also found that, below the Berezinskii-Kosterlitz-Thouless temperature $T_{B K T}$, the shape of the PDF of the magnetization is non-Gaussian and independent of the lattice size, in agreement with previous results [4]. However, we found that the shape of these distributions do depend on the temperature, contrarily to the generalized universality claimed in (BHP), who stated that the PDF of magnetization is independent of both, system size and temperature. Our results can be seen as a powerful extension of finite-size scaling and phenomenological renormalization of the PDF, suggested originally by Binder in the context of the Ising model [5], with sligth modifications introduced by the bounds of $M$. This allows in particular to understand the scaling form of the PDF of the order parameter in the $2 D X Y$-model as well as in the turbulent system.

The universality proposed by (BHP) might go beyond the idea of equivalence classes in Wilson's renormalization group approach [6], by including into a generalized universality class systems sharing the properties of finite size, strong correlations and self-similarity, even if their space-dimensions are different.

In [4, the two-dimensional probability distribution for the magnetization is calculated by means of a Monte Carlo simulation in the context of the $2 D H X Y$-model. This model is a further simplification of the Villain model [7], where the vortex variable $n$ is not a thermodynamical quantity, but it is constrained to the values $n=-1,0,1$. By using diagrammatic techniques, they showed that this asymmetry could be the result of three-spin interactions and higher order corrections.

Here, we consider the $2 D X Y$-model, which describes classical planar spins with nearest neighbor interactions, with a Hamiltonian given by 


$$
H=-J \sum_{\langle i, j\rangle} \cos \left(\theta_{i}-\theta_{j}\right)
$$

where $J$ is the ferromagnetic coupling constant and $\theta_{i}$ is the angle of orientation of the unitary spin vector $\vec{s}_{i}$. The summation $\langle i, j\rangle$ is over nearest neighbors and the spins are defined on the sites of a square lattice of lattice size $L$, with periodic boundary conditions. From hereon the ratio $k_{B} / J$ is set equal to unity throughout the paper. This model undergoes a remarkable binding-unbinding topological phase transition, such that the free energy and all its derivatives remain continuous [8], and no long-range order at low temperatures exists, as stated by the Mermin-Wagner theorem [9]. This model has been extensively studied through both numerical and analytical methods [10.

Our simulation was performed on a square lattice of lattice sizes $\mathrm{L}=10,12,16,22$, and 32 respectively. We estimate the $\mathrm{MC}$ sweeps needed for thermalization by plotting some observables like magnetization and energy. Typically $10^{5} \mathrm{MC}$ sweeps were used to reach thermal equilibrium. For thermal averages we used $5 \times 10^{5}$ spin configurations $\alpha_{j}$. Because the $2 D X Y$-model has a continuous line of critical points below $T_{B K T}$, special care was taken to choose statistical independent configurations to evaluate thermal averages of physical observables $X$. This was achieved by computing its normalized autocorrelation function [11]

$$
C(K)=\frac{<X_{\alpha_{i}} X_{\alpha_{i+K}}>-<X_{\alpha_{i}}><X_{\alpha_{K}}>}{<X_{\alpha_{i}}^{2}>-<X_{\alpha_{i}}>^{2}}
$$

where $X_{\alpha_{i}}$ is the value of $X$ in the configuration $\alpha_{i}$ at the $i$-th step along the MC-path through the configuration space, and the average $\langle\ldots\rangle$ was taken over this particular path of configurations separated by $K$ steps from each other. $C(K)=1$ for $K=0$, but for large enough $K, C(K)$ drops to zero, which means that these configurations become totally uncorrelated. We choose $K$ so that $C(K)$ was less that the recommended value 0.05 12. It is well known that as a critical system approaches the critical temperature, the decorrelation time $\tau$ diverges with the power law $\tau \sim \xi^{z}$, where $\xi$ is the (divergent) correlation length of the system and $z$ is known as the dynamical critical exponent, which is approximately two for local-flip algorithms like the Metropolis algorithm. This phenomenon is known as critical slowing down 13. This means in practice a serious limitation to numerical simulations of critical systems close to a critical point.

In fig. 1 we show MC data for the susceptibility for $L^{2}=256$ spins, as a function of the temperature. The peak occurs at the value $T_{C}(16)=1.15$, and corresponds to the temperature at which the correlation length equals $\mathrm{L}$, which is the standard definition of the critical temperature of a finite system. We compute also the errors (standard deviations), which become larger as the critical temperature is approached. Another interesting feature of these errors is that they are larger below $T_{C}$. This can probably be explained because of the comparative larger correlations lengths in this region, which corresponds to a continuous line of critical points with temperature dependent exponents [14] in the infinite volume limit.

We computed the critical temperature for the lattice sizes $\mathrm{L}=10,12,16,22,32$. For $\mathrm{L}=32$ we found an effective transition temperature $T_{C}=1.08$, in agreement with the value obtained by [15], where the linearized RG equations for the finite size scaling were used. Fig. 2 shows $T_{C}(L)$ as a function of $(\ln (L))^{-2}$. The values can be described by the finite size scaling formula [3] 


$$
T_{c}(L) \approx T_{\infty}+\frac{\pi^{2}}{4 c(\ln L)^{2}}
$$

where $T_{\infty}$ is the extrapolated value of the critical temperature for infinite volume. Within a few percents of error, we found that the value of $T_{\infty}$ agrees with the seemingly exact value 0.892 of the critical temperature $T_{B K T}$ of the Berezinskii-Kosterlitz-Thouless phase transition.

The ratio of the mean magnetization to critical temperature is plotted in fig. 3 as a function of $\ln (L)$. These values are compatible with a negative straight line, as suggested by [4] in the context of the harmonic XY-model. The values closer to the origin have larger statistical errors probably because of the finite size effects, which are proportional to $\ln (L)$ [3. 16].

Finally it should be emphasized that we did not make use of scaling relations to define physical quantities, like the shifted BKT-temperature $T^{*}(L)$, or temperature at which the renormalized spin-wave stiffness becomes the universal value $2 / \pi$ of the infinite system. This is because the use of the BKT theory beyond its confirmed validity needs at least justification, (the scaling region is defined by the inequality $\mid T-T_{B K T}$ ) $/ T_{B K T} \mid<10^{-2}$, where the renormalization group equations confidently apply [17]). In fact, we were able to obtain accurate values for thermal averages and test the scaling equations (see eqn. (3) and Figs. 2 and 3), in spite of the difficulties of numerical simulations due to the essential finite-size effects present in this model [3] $(\ln (L)$ dependence of physical quantities), and the very narrow critical region.

The renewed interest in the PDF of fluctuations of magnetization, $M$, is a consequence of the observation that similarly shaped PDF arise in completely different systems. For instance, in [2] it was found that fluctuations of the injected power in a confined turbulent flow show the same behavior. In Fig. 4 a plot of $\sigma Q(M)$ as a function of $(M-<M>) / \sigma$ can be seen, for lattice sizes $L=16$ and $L=32$ at the same temperature $T=0.70$. Here, $Q$ is the PDF of $M$ and corresponds to $P_{L}(M)$ in the language of Binder (see discussion below eqn. (4)). These curves have similar shapes like those found in turbulence experiments, but only within a reduced range of temperatures below $T_{B K T}$. These PDFs can be conveniently compared with the universal form $\Pi(y)=K\left(e^{b(y-s)-e^{b(y-s)}}\right)$ proposed in [1], by plotting the ratio $\sigma Q(M ; T) / \Pi(M)$ vs. $(M-\langle M\rangle) / \sigma$. In Fig. 5, four of such plots are displayed for $T=0.40,0.80,0.95$ and 1.05. The upper curves are successively multiplied by factors of 10 for clarity. As can be seen, when $T$ is increased, these ratios consistently change, showing the dependence of $Q(M ; T)$ on the temperature. The rightmost part of the lower curves is raised, probably due in part to the upper bound $M=1$. On the other hand, for $T /=0.95$ we can see that the opposite occurs. This temperature is slightly greater than $T^{*}$, above which the population of spin vortex pairs begins to increase. This happens because the system volume occupied by these vortices no longer contributes to the magnetization, which leads to a depleted probability density. This can be appreciated in the central and rightmost parts of the two upper curves. The hills are due to the bounds in the magnetization $0<M<1$. At $T=1.05$ this effect is greatly enhanced, and the leftmost part of the curve shows even more clearly the effect of the lower bound $M=0$. Concerning turbulent flows, we do not expect this type of bounding effects in the statistics of injected power. In principle, there are no limits to the fluctuations of such a quantity, and negative values are not excluded, meaning that the flow is delivering power to the driving system. Although this type of events are 
expected to be very unlike, they are not forbidden.

The temperature dependence of the PDF is not a surprising result. In fact, the use of the probability distribution of the order parameter to study finite size scaling and phenomenological renormalization, has been discussed by Binder in the context of the Ising model [18. For the region $\xi \sim L$ he proposed that the probability distribution function $P_{L}(M)$ does not depend separately on the three variables $\xi, L, M$, but only on the two scaled combinations, $L / \xi$ and $M \xi^{\beta / \nu}$ :

$$
P_{L}(M)=\xi^{\beta / \nu} \widetilde{P}\left(L / \xi, M \xi^{\beta / \nu}\right)=L^{\beta / \nu} P\left(L / \xi, M L^{\beta / \nu}\right) .
$$

He also argued that in the critical region $\xi \gg L, P_{L}(M)$ is no longer Gaussian. In the scaling region, it is a good approximation to take $P_{L}(M)$ equal to the PDF proposed by Bramwell et al. (The standard deviation $\sigma$ plays the role of $L^{-\beta / \nu}$ in the BHP distribution; this can be seen by using the standard definitions of the critical exponents and the relation $\left.\sigma=\sqrt{\frac{T}{L^{2}} \chi}\right)$. Nevertheless, and away from the region defined by $\left|T-T_{B K T}\right| / T_{B K T}<10^{-2}$, there is a temperature dependence in expresion (4) via the correlation length $\xi$ for finite size. It turned out that this dependence is rather weak in the range $0.5<T<T^{*}(L)$ $\left(T^{*}(L=16) \sim 0.94\right)$, but out of this range this dependence becomes stronger due to the presence of vortices and/or bounds.

In conclusion, we found that the probability distribution function for the magnetization is indeed independent of the system size, but its shape happens to vary with the temperature of the system, contrarily to the generalized universality proposed by Bramwell et al. [1]. This effect comes from the intrinsic temperature dependence on the first scaled variable $L / \xi$ in the distribution function proposed by Binder for the order parameter $M$. Also, there is a contribution coming from the constrained character of the magnetization.

We thank L. Vergara for helpful suggestions. This work was supported in part through projects FONDECYT 1980608 and 1990169, and DICYT 04-9631PA and 04-9631LM. 


\section{REFERENCES}

[1] S.T. Bramwell, P.C.W. Holdsworth and J.-F. Pinton, Nature 396 (1998) 552.

S.T.Bramwell et al., Phys. Rev. Lett. 84 (2000) 3744.

[2] R. Labbé, J.-F. Pinton and S. Fauve,Journal de Physique II France, 6 (1996) 1099.

[3] S.G. Chung, Phys. Rev. B 60, (1999), 11761.

[4] P. Archambault, S.T. Bramwell, P.C.W. Holdsworth, J. Phys. A:Math. Gen. 30 (1997) 8363.

P. Archambault,S.T. Bramwell,J.-Y. Fortin, P.C.W. Holdsworth, S. Peysson and J.-F. Pinton, J. Appl. Phys. 83 (1998) 7234.

[5] K. Binder, Computational Methods in Field Theory, eds. H. Gauslever and C.B. Lang, Springer (Berlin), (1992).

[6] K.G. Wilson and J. Kogut, Phys. Report 12C, (1974) 75.

K.G. Wilson, Rev. Mod. Phys. 47, (1975) 773.

[7] J. Villain, J. Physique 36, (1975) 581.

[8] V.L. Berezinskii, Sov. Phys. JETP 32 (1971) 493.

J.M.Kosterlitz and D.J.Thouless, J. Phys. C: Solid State Phys. 6 (1973) 1181-1203.

[9] N. D. Mermin and H. Wagner, Phys. Rev. Lett. 17 (1966) 1133.

[10] J. Tobochnik and G.V. Chester, Phys. Rev. B20 (1979) 3761.

R. Gupta and C.F. Baillie, Phys. Rev. B45 (1992) 2883.

J.R. Lee and S. Teitel, Phys. Rev. B46 (1992) 3247.

M. Campostrini, A. Pelisetto, P. Rossi and E. Vicari, Phys. Rev. B 54 (1996) 7301.

Z. Gulácsi and M. Gulácsi, Advances in Physics 47, (1998) 1-89.

J.V. José,L.P. Kadanoff, S. Kirkpatrick and D.R. Nelson, Phys. Rev. B16, (1977) 1217.

[11] J. J. Binney, N. J. Dowrick, A. J. Fisher and M. E. J. Newman, The Theory of Critical Phenomena, Oxford Science Pub. (1993).

[12] A. Sokal, Nucl. Phys. B 20 (Proc. Suppl.) (1991), 55.

[13] R. Swendsen and J.-S. Wang, Phys. Rev. Lett., 58, (1987) 86.

U. Wolff, Nucl. Phys. Proc. Suppl. 17 (1990) 93.

A. Sokal, Nucl. Phys. Proc. Suppl. 20 (1991) 55.

[14] J. Cardy, Scaling and Renormalization in statistical physics, Cambridge University Press, (1996).

C. Itzikson and J-M.Drouffe, Statistical Field Theory, Vol.1,Cambridge Monographs on Mathematical Physics, (1992).

[15] S.T. Bramwell and P.C.W. Holdsworth, J. Phys. : Condens. Matter 5, (1993) L53.

[16] M.Hasenbusch and K.Pinn, J.Phys. A: Math Gen. 30 (1997) 63.

R. Kenna and A. C. Irving, Phys. Lett., B 351 (1995) 273.

J.-K. Kim, Adauto F. De Souza, D. P. Landau, F. Phys. Rev. E 54 (1996) 2291.

W. Janke, Phys. Lett. A 173 (1993) 8.

[17] J. Cardy, Phys. Rev. B26, (1982) 6311.

[18] K. Binder, Z. Phys. V43 (1981) 119.

K. Binder, Phys. Rev. Lett. 47 (1981) 693. 


\section{Figure captions}

Figure 1:Susceptibility for $L=16$ in the range $0<T \leq 3$. The peak at 1.15 corresponds to the shifted critical temperature.

Figure 2: The shifted critical temperature for different lattice sizes is plotted as a function of the system size.

Figure 3: Scaling relation for the magnetization-temperature ratio as a function of system size.

Figure 4: Plots of $\sigma Q(M)$ vs. $(M-<M>) / \sigma$ at $T=0.70$ for lattice sizes $L=16(+)$ and $L=32(*)$.

Figure 5: $\sigma Q(M) / \Pi(M)$ ratios for four values of temperature (see text). 
Finite size scaling in the $2 \mathrm{~d} X Y$-model . . .

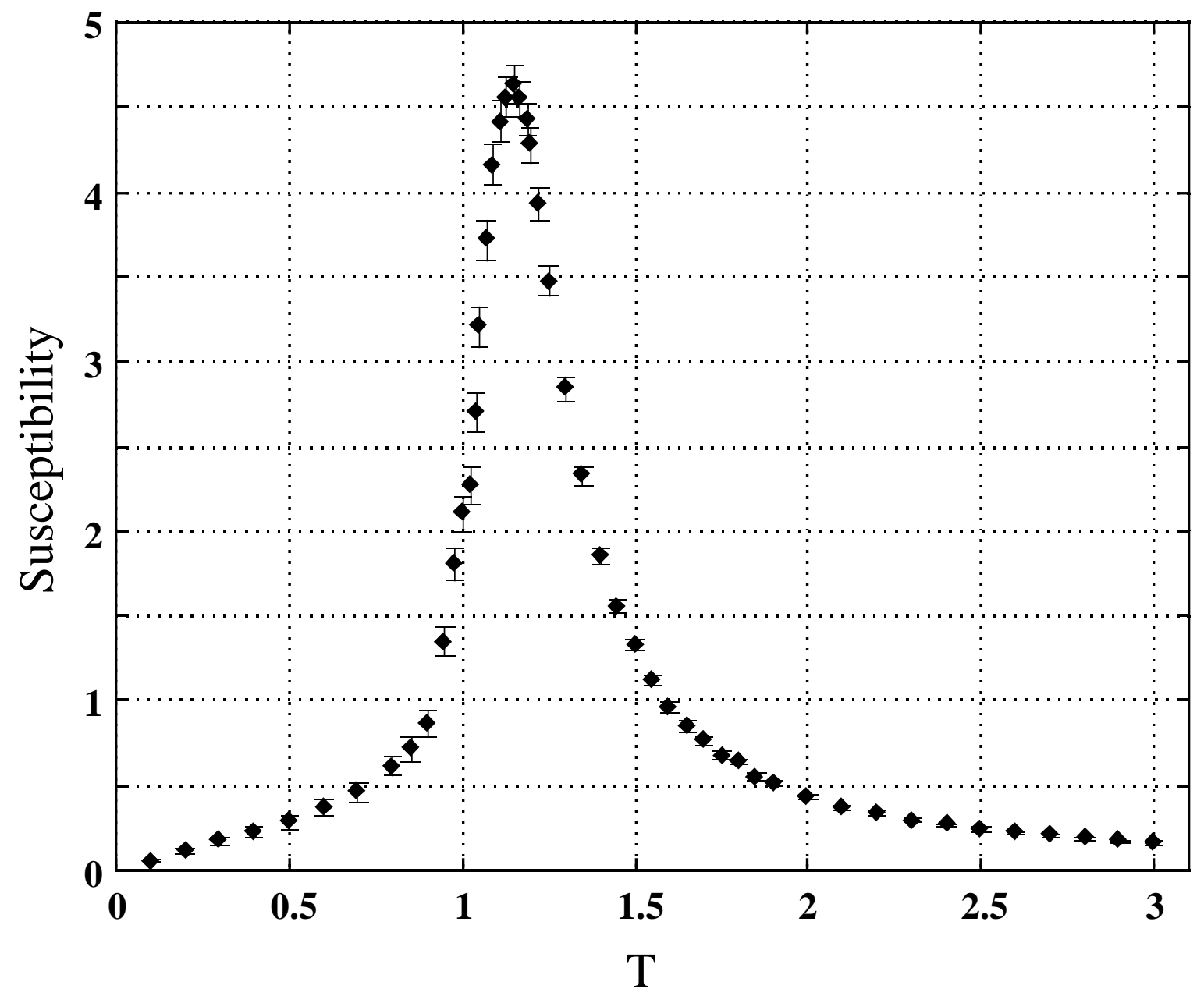


Finite size scaling in the $2 \mathrm{~d} X Y$-model . . . - Palma

FIGURE_2

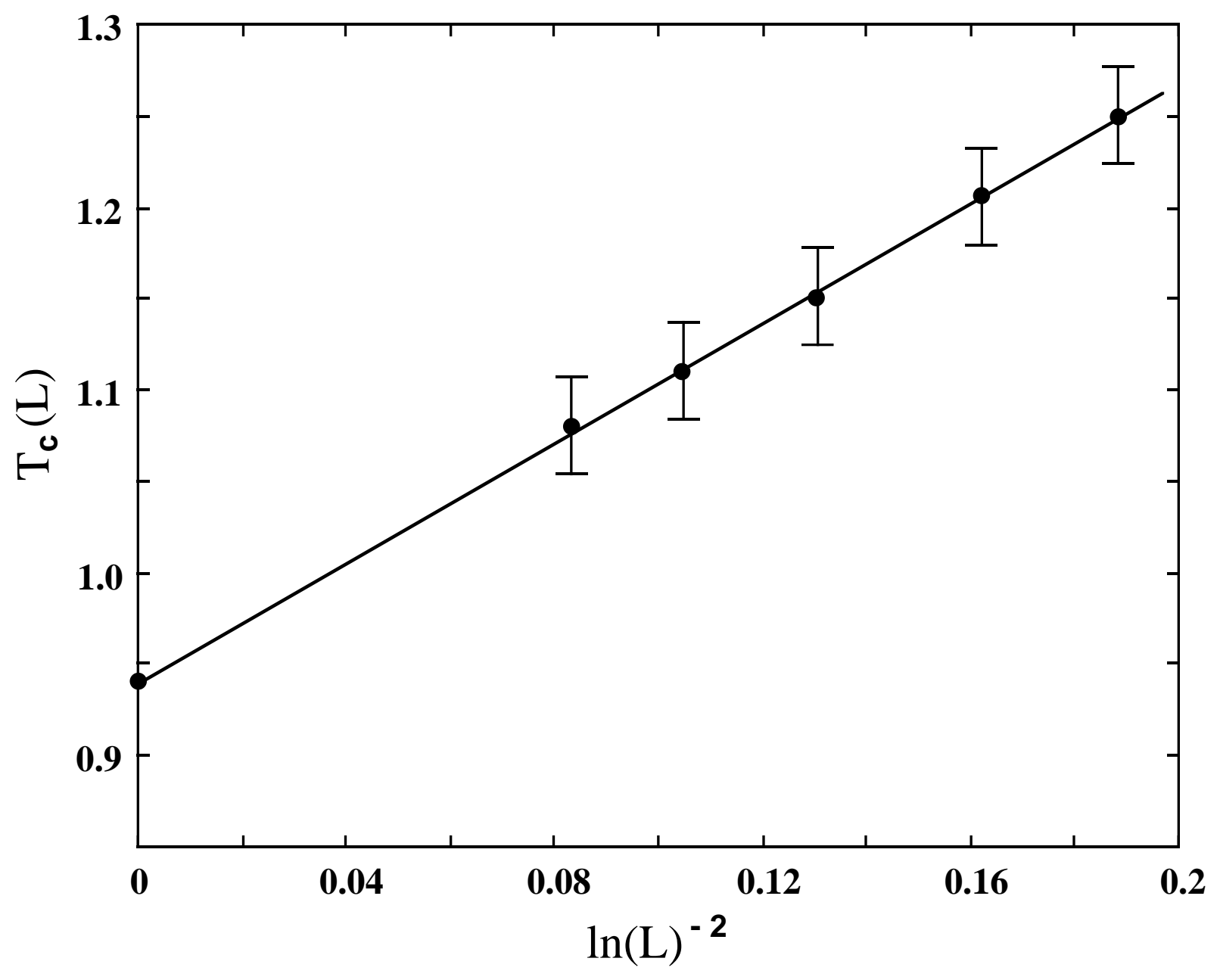


Finite size scaling in the $2 d X Y$-model . . . - Palma

FIGURE_3

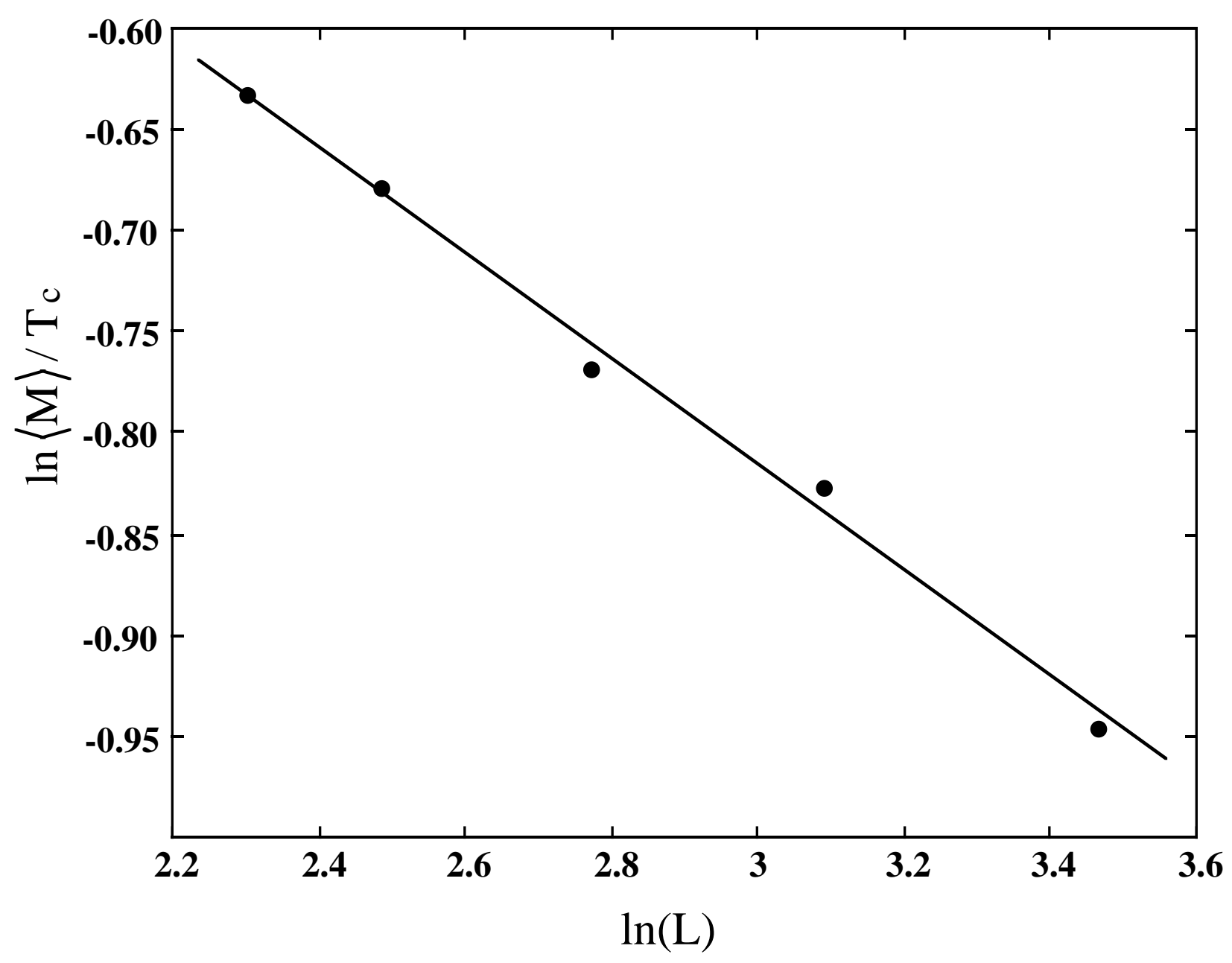


Finite size scaling in the $2 \mathrm{~d} X Y$-model . . . - Palma

FIGURE_4

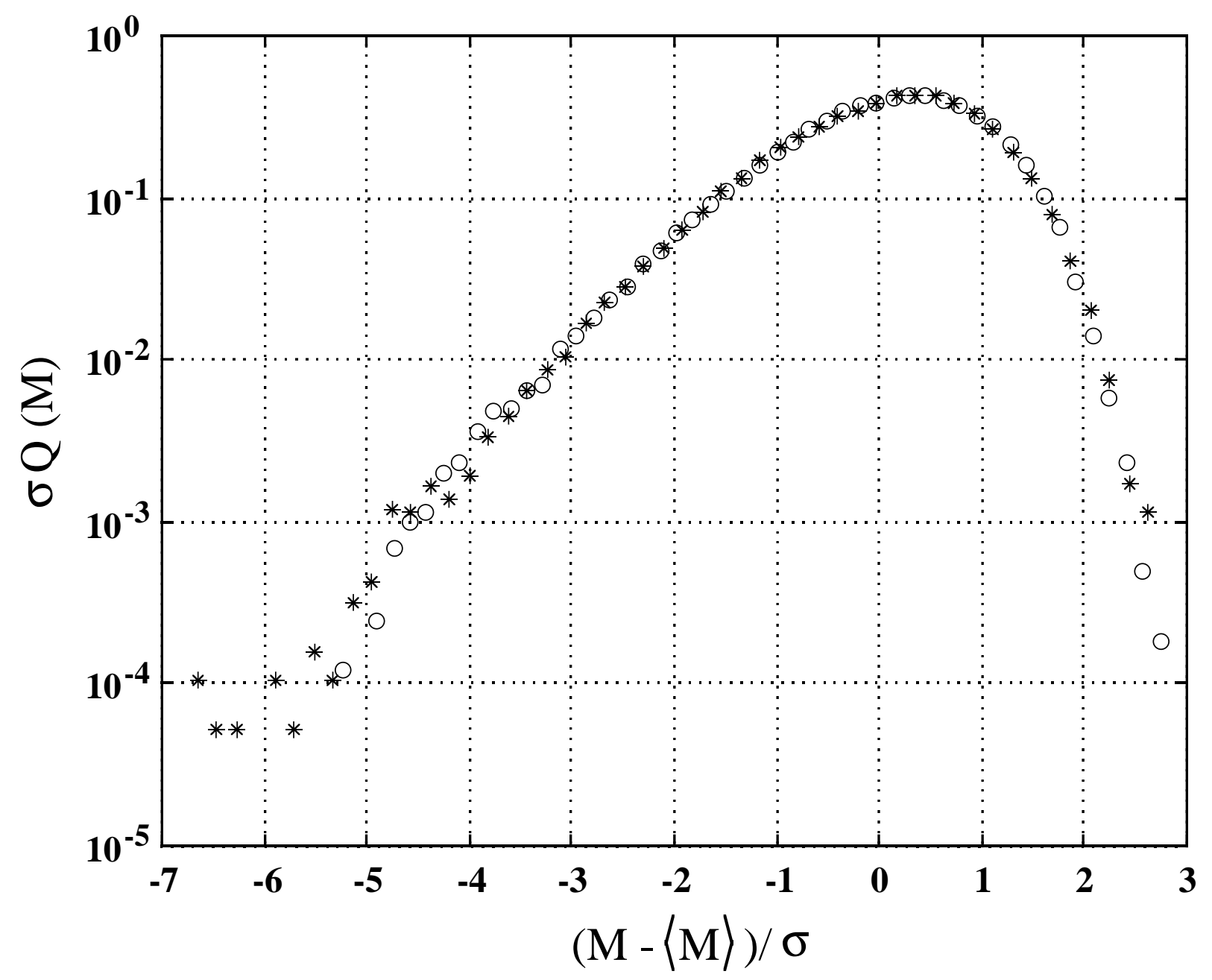


Finite size scaling in the $2 d X Y$-model . . . - Palma

FIGURE_5

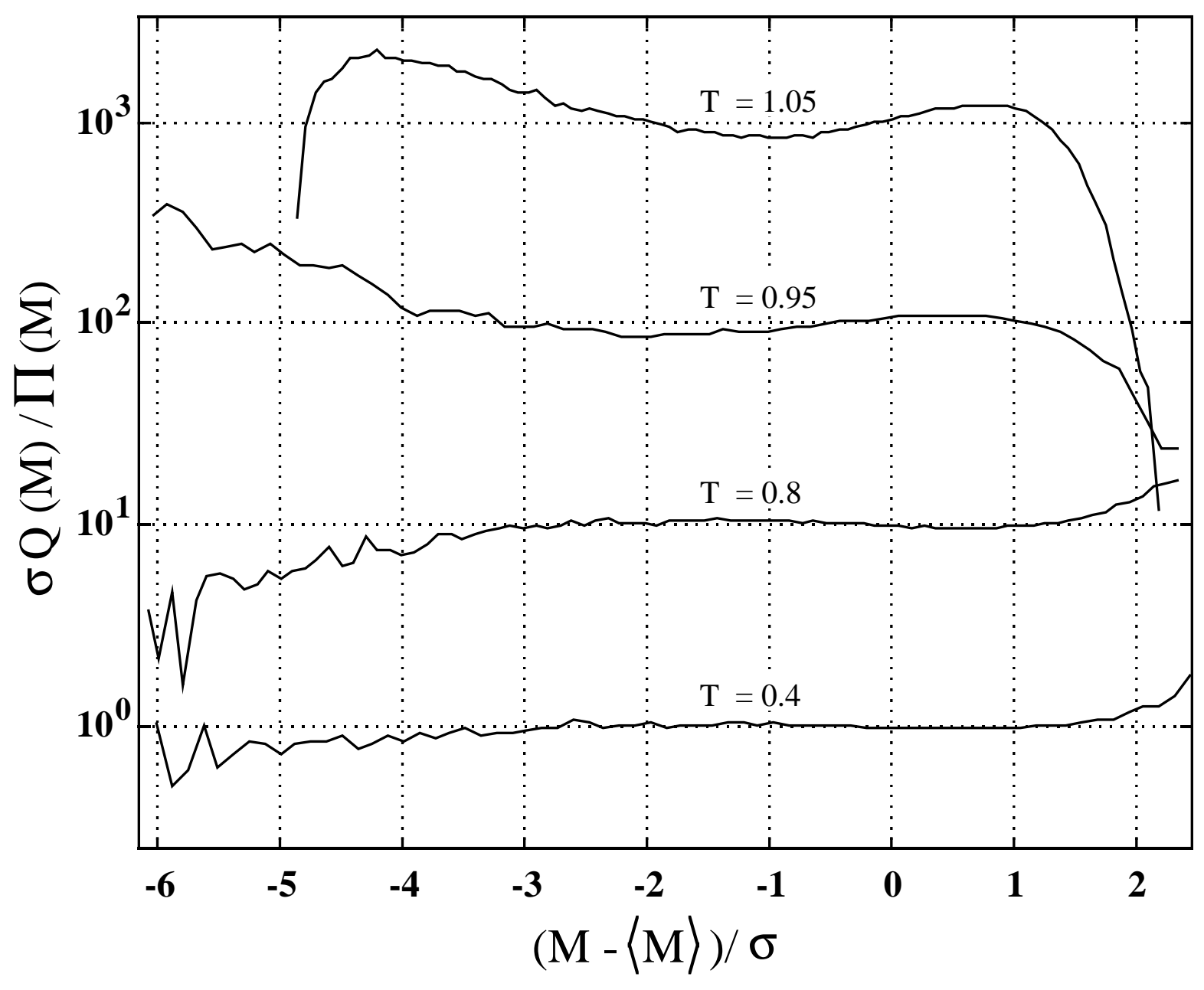

